replaces that of the circular sections. Such a surface may collapse into a confocal surface by a suitable strain, which in general differs from that required for circular sections. The case of the hyperbolic paraboloid can be regarded from either point of view, if its generators are regarded as circles of infinite radius.

\title{
Note on an alternant suggested by statistical theory.
}

\author{
By B. Babington Smith.
}

The determinant to be described made its appearance in the course of a search for the frequency distribution of Spearman's rank correlation coefficient (2). This name is given to the form, $\rho=1-\frac{6 \Sigma d^{2}}{n^{3}-n}$, taken by the product moment correlation coefficient in the special case where the two variables are separate arrangements of the first $n$ natural numbers.

For any value of $n$ the distribution of $\rho$ is completely determined by the values of $\Sigma d^{2}$, the sum of the squares of the differences between the $n$ pairs of numbers.

Consider the array

\begin{tabular}{l|rrrrrrr}
\multicolumn{1}{c}{} & 1 & 2 & 3 & $\ldots$ & $n-2$ & $n-1$ & $n$ \\
\hline & 0 & 1 & 2 & $\ldots \ldots$ & $n-3$ & $n-2$ & $n-1$ \\
2 & -1 & 0 & 1 & $\ldots \ldots$ & $n-4$ & $n-3$ & $n-2$ \\
$\ldots \ldots$ & $\ldots \ldots \ldots$ & $\ldots \ldots$ & $\ldots \ldots \ldots \ldots \ldots$ & $\ldots \ldots$ \\
$n-2$ & $-(n-3)$ & $\ldots \ldots$ & 0 & 1 & 2 \\
$n-1$ & $-(n-2)$ & $\ldots \ldots$ & -1 & 0 & 1 \\
$n$ & $-(n-1)$ & $\ldots \ldots$ & -2 & -1 & 0.
\end{tabular}

Any expression made up of $n$ entries from the body of this array chosen so that no row or column contributes more than one must correspond to a possible pairing of the numbers $l$ to $n$ with another arrangement of them. Further there are $n$ ! possible ways of choosing such expressions corresponding to the $n$ ! possible arrangements.

If now we write

$$
\begin{aligned}
& \begin{array}{lllll}
a^{0} & a^{12} & a^{22} & \ldots & a^{(n-1)^{2}}
\end{array} \\
& \begin{array}{lllll}
a^{12} & a^{0} & \ldots & \ldots & a^{(n-2)^{2}}
\end{array}
\end{aligned}
$$

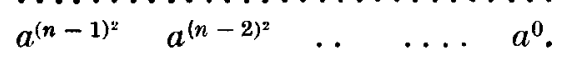


The indices of the $n$ ! expressions consisting of the products of $n$ entries (chosen so that each column and each row is represented once) give the $n !$ possible values of $\Sigma d^{2}$ and the coefficients of the collected terms will give the frequency distribution.

M. G. Kendall showed that the value $\Delta$ of the determinant of this array is

$$
\left(1-a^{2}\right)^{n-1}\left(1-a^{4}\right)^{n-2}\left(1-a^{6}\right)^{n-3} \ldots\left(1-a^{2(n-1)}\right) .
$$

This result may be reached in a variety of ways of which two appear to be of interest.

(i) Professor Turnbull has pointed out that

$$
\text { the operations } \quad \begin{array}{ll}
a^{(n-1)^{2}-(i-1)^{2}} & \times \text { column } i \\
a^{-(n-j)^{n}} & \times \operatorname{row} j
\end{array}
$$

give

$$
\left.a^{n(n-1)(n-2) / 3} \Delta=\begin{array}{lllll}
1 & 1 & \ldots & 1 & 1 \\
a^{2(n-1)} & a^{2(n-2)} & \ldots & a^{2} & 1 \\
a^{4(n-1} & a^{4(n-2)} & \ldots & a^{4} & 1
\end{array}\right]
$$

This is an alternant

$$
\begin{aligned}
& =\left(1-a^{2(n-1)}\right)\left(1-a^{2(n-2)}\right) \ldots\left(1-a^{2}\right) a^{2} a^{4} \ldots a^{2(n-2)} \\
& \left(1-a^{2(n-2)}\right) \ldots\left(1-a^{2}\right) a^{2} a^{4} \ldots a^{2(n-3)} \\
& \left(1-a^{2}\right) \\
& =\left(1-a^{2(n-1)}\right)\left(1-a^{2(n-2)}\right)^{2} \ldots\left(1-a^{4}\right)^{n-2}\left(1-a^{2}\right)^{n-1} \cdot a^{2 n(n-1)(n-2) / 6}
\end{aligned}
$$

and $\Delta$ has the value given.

By the same method

the determinant

$$
\begin{array}{cccccc}
1 & a & a^{2} & a^{3} & \ldots & a^{n-1} \\
a & 1 & a & a^{2} & \ldots & a^{n-2} \\
\ldots \ldots & \ldots \ldots & \ldots & \ldots & \ldots & \ldots \\
a^{n-1} & a^{n-2} & a^{n-3} & a^{n-4} & \ldots & 1
\end{array}
$$

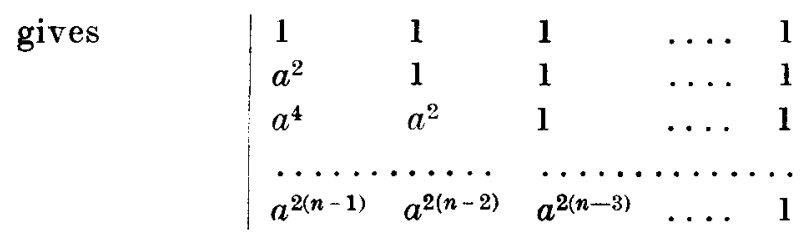

and this, by the operations row $_{j}-$ row $_{j+1}$,

$$
\left(1-a^{2}\right)^{n-1}
$$


ii. Dr Rutherford suggested the use of Rev. C. L. Dodgson's method of condensation ${ }^{1}$. Thus from

$$
\begin{array}{ccccccc}
1 & a & a^{4} & a^{9} & \ldots & a^{(n-1)^{2}} \\
a & 1 & a & a^{4} & \ldots & a^{(n-2)^{\prime}}
\end{array}
$$

the lst condensation

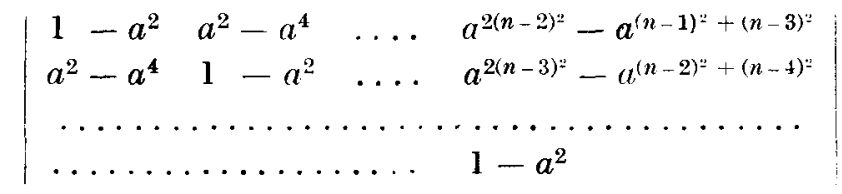

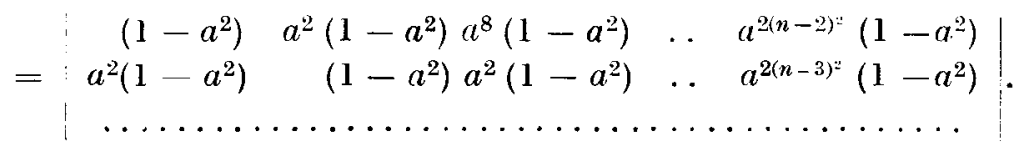

The 2nd condensation gives

$$
\begin{aligned}
& \frac{\left(1-a^{2}\right)^{2}-a^{4}\left(1-a^{2}\right)^{2}}{1} \quad \frac{a^{4}\left(1-a^{2}\right)^{2}-a^{8}\left(1-a^{2}\right)^{2}}{a} \cdots \\
& \frac{a^{4(n-3)^{2}}\left(1-a^{2}\right)^{2}-a^{4(n-2)^{2}+4(n-4)^{\prime \prime}}}{a^{(n-3)^{\prime \prime}}} \\
& \left(1-a^{2}\right)^{2}\left(1-a^{4}\right) \quad a^{3}\left(1-a^{2}\right)^{2}\left(1-a^{4}\right) \quad \ldots \quad a^{3(n-3=}\left(1-a^{2}\right)^{2}\left(1-a^{4}\right)
\end{aligned}
$$

The 3rd

$$
\begin{aligned}
& {\left[\begin{array}{c}
\left(1-a^{2}\right)^{4}\left(1-a^{4}\right)^{2}\left(1-a^{6}\right) \\
1-a^{2} \\
\ldots \ldots \ldots \ldots \ldots \ldots \ldots \ldots \ldots \ldots \ldots \ldots \ldots \ldots \ldots \ldots \ldots \ldots \ldots \ldots \ldots \ldots \ldots
\end{array}\right.}
\end{aligned}
$$

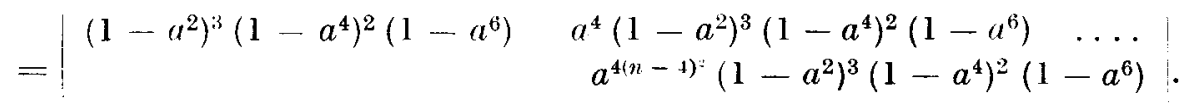

The rule of formation of terms in successive condensations now becomes clear and the final result will be as shown earlier.

This method, like the former, evaluates the determinant

$$
\begin{aligned}
& 1 \quad a \quad a^{2} \quad a^{3} \quad \ldots \quad a^{n-1} \\
& a^{n-1} \quad \ldots \ldots \ldots \ldots \ldots \ldots \ldots
\end{aligned}
$$

but similiar expressions with other powers of $a$ seem intractable. It should be remarked that it is the permanent of the original matrix which after all yields the frequency distribution required since this 
xxii

is based on the number of occurrences of a power of $a$ without regard to sign.

Three valuable properties of this permanent are ${ }^{2}$

(a) that it may be expanded in terms of its minors

(b) that any minor, when expanded, is a "reciprocal" polynomial in $a$

(c) the effect of shifting a minor bodily across it is to multiply each term of its expansion by a constant power of $a$.

This determinant does not appear to have been noted previously.

Department of Psychology,

UNIVERSITY OF ST ANDREWS.

\section{REFERENCES.}

1 Dodgson, Rev. C. L., "On condensation of determinants." Proc. Roy. Soc., Lond., xv., 150 .

2 Kendall, M. G., Kendall, S. F. H. and Babington Smith, B., "'The distribution of Spearmans coefficient of rank correlation ....." Biometrica, xxx., Pts. iii. and iv., p. 251.

\section{Maxima and minima}

By G. Lawson,

In my experience answers to questions about maxima and minima are as a rule hazy and unsatisfactory. I suggest that the principle "according as a function is increasing or decreasing, its derivative is positive or negative" might be applied more fully. All functions are here assumed continuous.

If $y$ is a maximum at $A$, then

$$
\text { before } A \quad \text { at } A
$$

(1) $y$ is increasing

(4) $y_{1}$ is + , by (1)

$$
\text { (3) } y \text { is maximum }
$$

(6) $y_{1}=0$, by (4) and (5) after $A$

(2) $y$ is decreasing

(5) $y_{1}$ is - , by (2) 